The entire model with a full set of replaceable parts (initial start-up costs) sells for $\$ 6,700$. For vaginal hysterectomy, we have two options for parts to use. Our premium vaginal hysterectomy set (the high-fidelity cartridges we used for the study) retail for a combined $\$ 440$. The premium vagina costs $\$ 270$ and the premium uterus costs $\$ 170$. After the hysterectomy is performed, the premium vagina can be used for a variety of other procedures including transobturator slings, retropubic slings, anterior colporrhaphy, posterior colporrhaphy, and sacrospinous ligament suspension. Average cost for these procedures is $\$ 45.00$. The uterus can be repurposed to create fibroids to practice operative hysteroscopy bringing the cost per procedure to $<\$ 85$. So, while the initial cost for a vaginal hysterectomy with the premium vaginal hysterectomy set is $\$ 440$, the cost per procedure can be greatly reduced with the parts being used for other procedures. We also offer our training uterus and vagina for a combined cost of $\$ 218$. The training uterus sells for $\$ 99$ and the training vagina sells for $\$ 119$.

The premium vagina and premium uterus support the following procedures:

- Vaginal Hysterectomy with bleeding

- Anterior Colporrhaphy

- Posterior Colporrhaphy

- Transobturator Sling

- Retropubic Sling

- Colpocleisis

- McCall's culdoplasty

The premium vagina contains the following features:

- Two distinct layers to support a full-thickness vaginal wall dissection

- Realistic cutting, suturing, and puncturing haptics

The premium uterus contains the following features:

- Anatomically correct shape with slight anteflexion

- Surgical tubing capable of hooking up to an IV system to simulate bleeding

- Mesh embedded in the ligament aspects of the uterus to support proper suture placement

- Endopelvic fascia marker to signify correct anterior cul de sac entry

The training vagina and training uterus support the following procedures:

- Vaginal Hysterectomy without bleeding

- McCall's culdoplasty

The training vagina contains the following features:

- Single layer in the vagina

- McCall's culdoplasty

The training uterus contains the following features:

- Anatomically correct shape with slight anteflexion

Chen CCG, Lockrow EG, Destephano C, Nihira MA, Matthews C, Kammire L, et al. Establishing validity for a vaginal hysterectomy simulation model for surgical skills assessment. Obstet Gynecol 2020;136.

The authors provided this information as a supplement to their article.

(C2020 American College of Obstetricians and Gynecologists.

Page 1 of 5 
1. Initial inspection (Check one)

2. Incision (Check one)

\section{Does not} perform appropriate incision(s) safely and does not use incision(s) effectively ensuring optimal exposure

3. Maintenance of visibility (Check one)

\section{Almost never or} never obtains appropriate exposure

4. Use of assistants (Check one)

5. Knowledge of
Almost never or never

strategically uses assistant(s) to the best advantage instruments (Check one) never uses and is familiar with correct instruments

6. Tissue and instrument
Almost never or

$\square_{1}$
Partially
complete and
unsystematic
inspection of
relevant pelvic
and vaginal
structures

$\square 1$ Incompletely performs appropriate incision(s) safely and does not use incision(s) effectively ensuring optimal exposure

\section{$\square 2$} Complete but unsystematic inspection of relevant pelvic and vaginal structures

$\square 2$

Performs appropriate incision(s) safely but does not use incision(s) effectively ensuring optimal exposure

$\square 1$
A few times (less
than half the
time) obtains
appropriate
exposure

\section{$\square$ 2}

Sometimes (about half the time) obtains appropriate exposure

$\square$
A few times (less
than half the
time)
strategically uses
assistant(s) to
the best
advantage

\section{$\square_{2}$}

Sometimes (about half the time)

strategically uses assistant(s) to the best advantage

$\square$ A few times (less than half the time) uses and is familiar with correct instruments

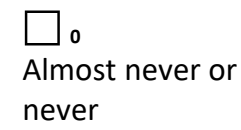

$\square_{3}$
Complete and
somewhat
systematic
inspection of
relevant pelvic
and vaginal
structures

$\square_{3}$

Performs appropriate incision(s) safely and partially uses incision(s) effectively ensuring optimal exposure

\section{$\square_{4}$}

Systematic and complete

assessment of relevant pelvic and vaginal structures
Not observed

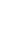


handling

(Check one)

appropriately

handles tissue

and instruments

7. Electro-

surgery

(Check one)

$\square$ 。

Almost never or

never uses

electro-surgery

safely and

efficiently

8. Knot tying

/ligation

(Check one)

9. Hemostasis (Check one)

Almost never or never exposes bleeders and uses correct technique to obtain hemostasis safely and effectively never quickly and correctly performs suture ligation and knot tying time) appropriately handles tissue and instruments

$\square$ A few times (less than half the time) uses electro-surgery safely and efficiently time) handles tissue and instruments appropriately

$\square 2$ Sometimes (about half the time) uses electro-surgery safely and efficiently time) handles tissue and instruments appropriately

$\square_{3}$ Most time (more than half the time) uses electro-surgery safely and efficiently tissue and instruments appropriately Almost always or always uses electro-surgery safely and efficiently
Not observed

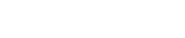

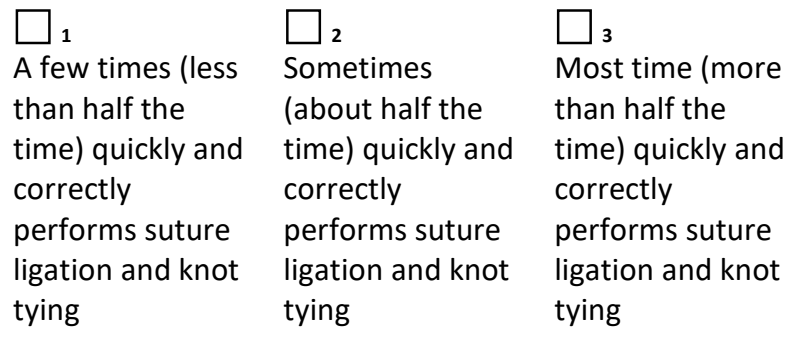

$\square$

Almost always or always quickly and correctly performs suture ligation and knot tying

\section{Not observed}




\begin{tabular}{|c|c|c|}
\hline $\begin{array}{l}\text { unnecessary } \\
\text { noves }\end{array}$ & $\begin{array}{l}\text { unnecessary } \\
\text { moves }\end{array}$ & $\begin{array}{l}\text { unnecessary } \\
\text { moves }\end{array}$ \\
\hline
\end{tabular}

12. Flow of operation and forward planning (Check one) $\square$ Almost never or never demonstrates forward planning allowing for proper flow of the procedure $\square_{1}$ A few times (less than half the time)

demonstrates forward planning allowing for proper flow of the procedure
Sometimes (about half the time)

demonstrates forward planning allowing for proper flow of the procedure $\square_{3}$ Most time (more than half the time) demonstrates forward planning allowing for proper flow of the procedure
Almost always or always demonstrates forward planning allowing for proper flow of the procedure
13. Knowledge of specific procedure (Check one)
Almost never or

never

demonstrates

familiarity with all aspects of the operation

$\square_{1}$
A few times (less
than half the
time)
demonstrated
familiarity with
all aspects of the
operation

$\square_{2}$
Sometimes
(about half the
time)
demonstrates
familiarity with
all aspects of the
operation

$\square 3$
Most time (more
than half the
time)
demonstrates
familiarity with
all aspects of the
operation

\section{$\square$} Almost always or always demonstrates familiarity with all aspects of the operation

Overall, is the trainee competent to safely perform this task unsupervised: NO YES Comments:

Reprinted from Chen C, Korn A, Klingele C, et al. Objective assessment of vaginal surgical skills. Am J Obstet Gynecol 2010;203:79.e1-8. Copyright 2010, with permission from Elsevier.

Chen CCG, Lockrow EG, Destephano C, Nihira MA, Matthews C, Kammire L, et al. Establishing validity for a vaginal hysterectomy simulation model for surgical skills assessment. Obstet Gynecol 2020;136.

The authors provided this information as a supplement to their article. 
Appendix 3. Receiver operating characteristic curve identifying a modified Vaginal Surgical Skills Index cutoff score of 27 (red arrow) as $80.3 \%$ sensitive (95\% Cl 69.0-89.0) and $96.7 \%$ specific (95\% Cl 88.0-99.5) for differentiating between competent and noncompetent surgeons at performing vaginal hysterectomy on a simulation model. Diagonal segments produced by ties.

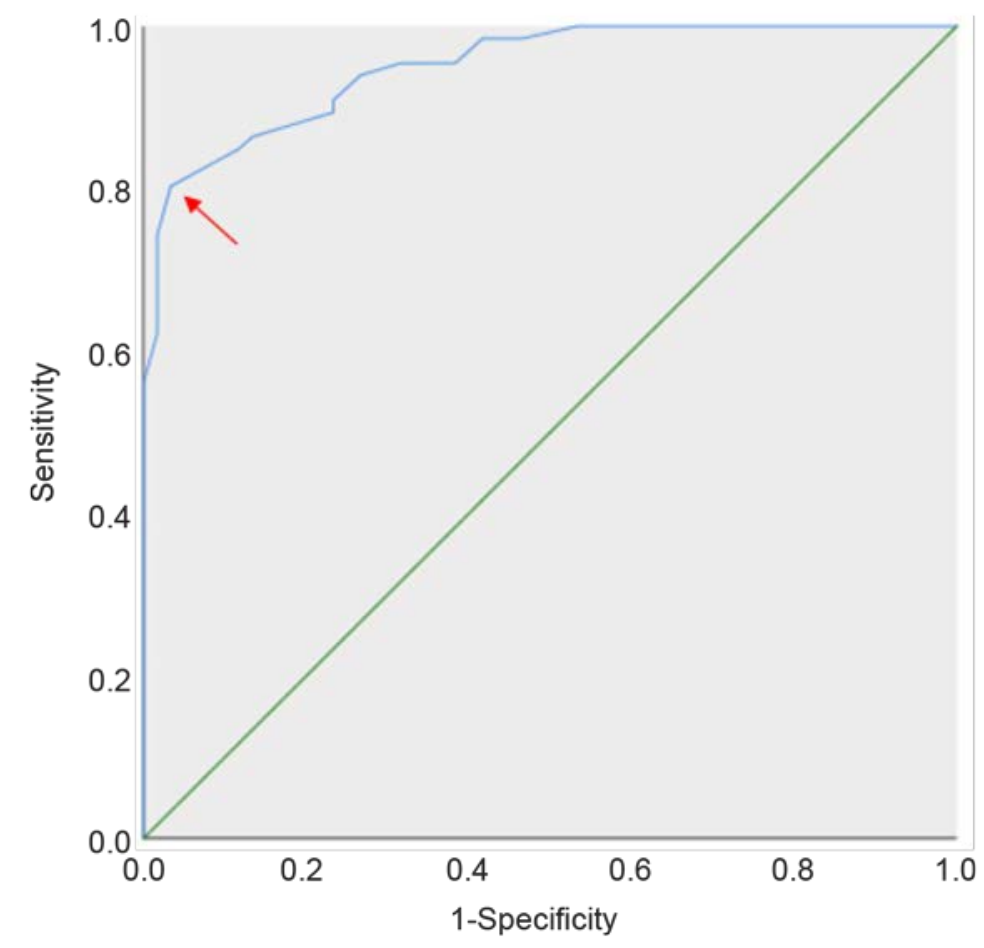

Chen CCG, Lockrow EG, Destephano C, Nihira MA, Matthews C, Kammire L, et al. Establishing validity for a vaginal hysterectomy simulation model for surgical skills assessment. Obstet Gynecol 2020;136.

The authors provided this information as a supplement to their article.

(C2020 American College of Obstetricians and Gynecologists.

Page 5 of 5 